\title{
Análisis experimental y teórico del ensayo de adherencia capa - sustrato en un acero DIN UC1 tratado termoquímicamente por borurización
}

\section{Experimental and theoretical analysis of the layer - substrate adhesion test on a DIN UC1 steel thermochemically treated by boronizing}

\author{
MARTÍNEZ-VÁZQUEZ, J. Merced†*, PÉREZ-PÉREZ, Arnulfo, RODRÍGUEZ-ORTIZ, Gabriel y \\ BAÑOS-LÓPEZ, Esperanza
}

Universidad Politécnica de Juventino Rosas, Ingeniería Metalúrgica. Hidalgo 102, Comunidad de Valencia, Santa Cruz de Juventino Rosas, Gto. 38253.

ID $1^{\text {er }}$ Autor: J. Merced, Martínez-Vázquez / ORC ID: 0000-0002-6230-3846, CVU CONACYT ID: 93450

ID $1^{\mathrm{er}}$ Coautor: Arnulfo, Pérez-Pérez / ORC ID: 0000-0001-6354-8899, CVU CONACYT ID: 176434

ID $2^{\text {do }}$ Coautor: Gabriel, Rodríguez-Ortiz / ORC ID: 0000-0002-3615-1973, CVU CONACYT ID: 48565

ID $3^{\text {er }}$ Coautor: Esperanza, Baños-López / ORC ID: 0000-0003-3983-4507, CVU CONACYT ID: 514562

DOI: $10.35429 /$ JSI.2020.14.4.7.14

Recibido Abril 13, 2020; Aceptado Junio 29, 2020

\section{Resumen}

En este trabajo se evaluó el efecto del tratamiento termoquímico de borurización sobre la adherencia y morfología superficial de la capa de boruro formada en el acero DIN UC1. El proceso se llevó a cabo por empaquetamiento a la temperatura de $1273 \mathrm{~K}$, a los tiempos de 4800, 6000, 7200 y 14400 segundos. La prueba de adherencia HRC basada en la norma VDI 1398, se simuló en COMSOL 5.0®; analizando el efecto del espesor de la capa de boruro y la rugosidad en la tensión de Von Mises, además de la tensión en la huella de indentación; en la cual se observó que al aumentar el espesor de la capa de 22.2 a $37.8 \mu \mathrm{m}$ las tensiones aumentaron, y por lo tanto mejoró la adherencia de la capa en el sustrato, lo cual ocasiona solo la formación de microgrietas. En cambio, en el espesor de capa mayor $(60.04 \mu \mathrm{m})$ la capa se delamina. Por lo tanto, para una aplicación industrial del acero DIN UC1 tratado termoquímicamente por borurización se recomiendan espesores de capa hasta $37.8 \mu \mathrm{m}$.

\begin{abstract}
In this work, the effect of the boronizing thermochemical treatment on the adherence and surface morphology of the boride layer formed in DIN UC1 steel was evaluated. The process was carried out by packing at the temperature of $1273 \mathrm{~K}$, at the times of 4800, 6000, 7200 and 14400 seconds. The HRC adherence test based on the VDI 1398 standard, was simulated in COMSOL 5.0®; analysing the effect of the thickness of the boride layer and the roughness on the Von Mises stress, in addition to the stress on the indentation footprint; in which it was observed that by increasing the thickness of the layer from 22.2 to 37.8 $\mu \mathrm{m}$ the stresses increased, and therefore the adhesion of the layer on the substrate improved, which causes only the formation of microcracks. On the other hand, in the greater layer thickness $(60.04 \mu \mathrm{m})$ the layer delaminates. Therefore, for an industrial application of DIN UC1 steel treated thermochemically by borurization, layer thicknesses up to $37.8 \mu \mathrm{m}$ are recommended.
\end{abstract}

\footnotetext{
* Correspondencia al Autor (Correo Electrónico: jmartinez_ptc@upjr.edu.mx)

$\dagger$ Investigador contribuyendo como primer autor.
} 


\section{Introducción}

La necesidad de mejorar las propiedades mecánicas de los aceros, ha impulsado el estudio de las técnicas de aplicación de recubrimientos, tratamientos térmicos y termoquímicos para aumentar su vida útil e incrementan la resistencia al desgaste.

Las fallas por desgaste, definidas como la pérdida de función de un elemento por deformación (fluencia) o separación de sus partes (fractura), son el resultado de la remoción o desplazamiento de material de la superficie de una pieza o componente a través del contacto y el movimiento relativo con un sólido, líquido o gas [1]; existe una influencia significativa de la fricción y la lubricación en el índice y severidad de los daños por desgaste [2]. En la resistencia al desgaste, se ha observado que la rugosidad y por consecuencia, el coeficiente de fricción por deslizamiento en el recubrimiento era extremadamente pequeño, por lo que el uso de un recubrimiento puede reducir sustancialmente el desgaste [3]. La pérdida de material reduce la capacidad de carga y la adherencia provocando un aumento en la fricción debido a la generación de residuos. Sobre la base de los datos limitados disponibles, existe información para suponer que la fricción por deslizamiento se relaciona con velocidades de desgaste más bajas debido al empleo de recubrimientos [4] en los que la adherencia juega un papel relevante.

Los tratamientos termoquímicos aportan propiedades para aplicaciones específicas como: disminuir el coeficiente de fricción, aumentar la resistencia a la corrosión y el desgaste. El obtener una óptima resistencia al desgaste [5], depende de la morfología de la superficie [6], la estructura física, la composición química y la rugosidad [7]. Estos tratamientos mejoran las propiedades tribológicas $[8,9,10]$, logrando extender la vida útil en las piezas de acero utilizadas en los sistemas mecánicos; lo que ha llevado a reconocer el valor del análisis de las superficies en donde se aplicó un tratamiento termoquímico [11].

Para predecir la falla se han desarrollado varias teorías basándose en observaciones experimentales [12].
Por ejemplo, la Teoría de la Energía de Distorsión fue propuesta por R. Von Mises [13] que observó que los materiales bajo esfuerzos hidrostáticos soportan esfuerzos mucho mayores que sus esfuerzos de fluencia bajo otros estados de carga [14]. Por lo que la distorsión del elemento es debida a los esfuerzos principales restándoles los esfuerzos hidrostáticos $\left(\sigma_{\mathrm{h}}\right)$, definidos como:

$\sigma_{h}=\frac{\sigma_{1}+\sigma_{2}+\sigma_{3}}{3}$

Donde $\sigma_{i}$ representa los esfuerzos por fluencia. La energía de distorsión es la diferencia entre la energía total de deformación por unidad de volumen y la energía de deformación por unidad de volumen debida a los esfuerzos hidrostáticos. Como el material se encuentra en el rango elástico (la falla se produce al llegar a la zona plástica), por lo tanto, el esfuerzo de von Mises se define como:

$$
\begin{aligned}
& \sigma^{\prime}=\sqrt{\sigma_{1}^{2}+\sigma_{2}^{2}+\sigma_{3}^{2}-\sigma_{1} \sigma_{2}-\sigma_{2} \sigma_{3}-\sigma_{1} \sigma_{3}} \\
& =\sqrt{\frac{\left(\sigma_{1}-\sigma_{2}\right)^{2}+\left(\sigma_{2}-\sigma_{3}\right)^{2}+\left(\sigma_{1}-\sigma_{3}\right)^{2}}{2}}
\end{aligned}
$$

Entonces la falla ocurre cuando:

$$
\sigma^{\prime}=S_{y}
$$
fluencia.

Donde $\mathrm{S}_{\mathrm{y}}$ el esfuerzo cortante a la

Se han presentado algoritmos iterativos que resuelven el desplazamiento y las tensiones inducidas por debajo de la superficie de una capa sólida elástica por un indentador rígido bajo condiciones de carga [15] utilizando el potencial de Lennard-Jones para modelar la fuerza de adhesión que actúa entre el penetrador y el sólido, mientras que los efectos de la adhesión a la superficie se caracterizan mediante el parámetro de Tabor. También se ha encontrado que el módulo de cizallamiento y el espesor del recubrimiento tienen un efecto significativo en el comportamiento del material; con recubrimientos más duros se experimentan tensiones de tracción significativamente más grandes, pero un menor desplazamiento de la superficie que en recubrimientos suaves. 
La presente investigación permite deducir que, a pequeñas escalas, la adhesión superficial es decisiva en la causa de desgaste o falla del material si los recubrimientos están mal diseñados. Este trabajo también demuestra que existe la necesidad de entender los efectos de la adhesión a la superficie en una escala pequeña para un buen diseño de los recubrimientos. Esto se debe a que cuando el contacto se carga y se descarga repetidamente, se observa una región de alto estrés a lo largo de los bordes de contacto que podrían iniciar el desgaste adhesivo como resultado de la fatiga, dando lugar a la pérdida de la protección. Los estudios de Microscopía de Fuerza Atómica (AFM), en donde se obtienen imágenes topográficas de diferentes porciones de la superficie son complementarias al estudio de adherencia, ya que la disminución en dureza y resistencia a la corrosión se puede deber a la degradación de la superficie del material a medida que aumenta la rugosidad.

\section{Metodología}

El tratamiento de borurización se realizó en el acero DIN UC1, la composición se muestra en la Tabla 1, por empaquetamiento en una mezcla de polvos compuesta por $30 \%$ en peso de ácido de ácido bórico, $30 \%$ en peso de bórax y el resto de carburo de silicio [16] a la temperatura de 1273 K. El proceso se llevó a cabo introduciendo los sustratos preparados metalográficamente, en una caja de $100 \times 150 \times 70 \mathrm{~mm}$ de placa de acero de 1/4. durante $2 \mathrm{~h}, 3 \mathrm{~h}$ y $4 \mathrm{~h}$. La caja de acero se colocó dentro de un horno NABERTHERM precalentado a la temperatura de los tratamientos.

\section{\begin{tabular}{l|l|llll|l|l}
$\mathbf{C}$ & $\mathbf{M n}$ & $\mathrm{Si}$ & $\mathbf{C r}$ & $\mathbf{C u}$ & $\mathbf{N i}$ & $\mathbf{A l}$ & $\mathbf{S}$
\end{tabular}}

\begin{tabular}{|l|l|l|l|l|l|l|l|}
\hline 0.53 & 0.74 & 0.30 & 0.20 & 0.21 & 0.082 & 0.024 & 0.008 \\
\hline
\end{tabular}

Tabla 1 Composición química del acero DIN UC1 (\% peso)

Fuente: elaboración propia [Word]

Los sustratos de acero DIN UC1 se desbastaron con lijas de carburo de silicio con tamaño de partícula 240, 320, 400, 600, 1000, 2000 por último se pulieron con paño de microfibra y alúmina de $0.05 \mu \mathrm{m}$. Al terminar el tratamiento las muestras fueron retiradas de la caja, enfriadas a temperatura ambiente, preparadas metalográficamente y atacadas con Nital al $3 \%$.

\section{Microscopía óptica}

En el estudio se utilizó un Microscopio Metalográfico marca Olympus con el software para el análisis de imágenes PaxIt, las micrografías se obtuvieron a 200X para corroborar la presencia de la capa de boruro y medir su espesor.

\section{Microscopía de Fuerza Atómica (AFM)}

La medición de la rugosidad se realizó utilizando un Microscopio de Fuerza Atómica Nanosurf® EasyScan 2 en modo contacto. Las pruebas se realizaron en áreas de $50 \times 50 \mu \mathrm{m}$, $25 \times 25 \mu \mathrm{m}$ y $5 \times 5 \mu \mathrm{m}$. donde se reportan los valores en nanómetros de la altura mínima, altura máxima, valor promedio, mediana, rugosidad promedio $\left(\mathrm{R}_{\mathrm{a}}\right)$ y raíz cuadrada de la $\operatorname{rugosidad}\left(\mathrm{R}_{\mathrm{ms}}\right)$.

\section{Prueba de adherencia}

La simulación se realizó de acuerdo con el método de ensayo de indentación DaimlerBenz Rockwell C con un indentador cónico de diamante con una punta de $200 \mu \mathrm{m}$ de radio y una carga de $150 \mathrm{~kg}$. Los diferentes tipos de daño observados sirvieron de comparación con los estados convencionales de calidad de adherencia de acuerdo con la escala HF (de HF1 a HF6) [17] que representa la fuerza de adherencia entre el sustrato y el recubrimiento. Entre HF1 y HF4 la adherencia es suficiente, mientras que HF5 y HF6 representan una adherencia insuficiente.

\section{Simulación en COMSOL®}

COMSOL Multiphysics ®es un software para el modelado de sistemas físicos basándose en el Método del Elemento Finito. El proceso se ejecutó a través de los siguientes pasos: la creación de una geometría, la especificación de una física, la construcción de un mallado, la elección del tipo de solución y la visualización de los resultados, utilizando el módulo de Mecánica Estructural 


\section{Resultados}

\section{Microscopía óptica}

La dinámica de crecimiento de la capa de boruro consta de tres etapas [18], durante la primera los cristales de $\mathrm{FeB}$ (el primer producto que se forma) creció sobre la superficie metálica hasta cubrir la pieza por completo. Durante la segunda etapa los cristales de FeB aumentaron y formaron $\mathrm{Fe}_{2} \mathrm{~B}$ que se desplazó hacia el interior del metal y en la tercera etapa todos los cristales de $\mathrm{Fe}_{2} \mathrm{~B}$ tuvieron una orientación cristalográfica preferente [001] dando lugar a morfología tipo diente de sierra [19] como se muestra en la Figura 1.

El espesor de la capa es un parámetro importante en las propiedades finales de la pieza, debido a la forma irregular propia del boruro de hierro la medición se realizó de acuerdo con el procedimiento descrito por Keddam y colaboradores [20] que consiste en realizar varias mediciones en distintos puntos del sustrato y posteriormente promediarlas.
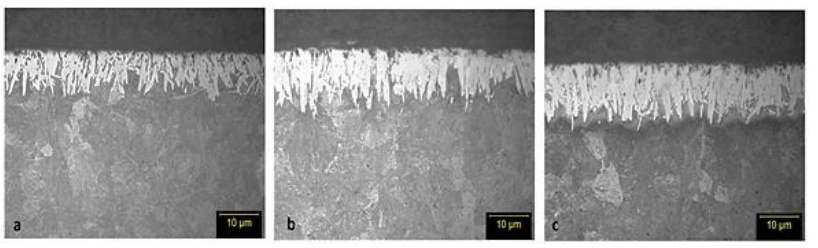

Figura 1 Micrografías a 200X de la capa de boruro de hierro $\left(\mathrm{Fe}_{2} \mathrm{~B}\right)$ a $1273 \mathrm{~K}$ durante 2, (b) 3 y (c) 4 horas Fuente: elaboración propia [PaxIt]

El crecimiento de espesor de la capa de boruro de hierro depende de la temperatura y el tiempo del tratamiento, es decir a mayor temperatura el espesor de la capa es más grande, como se muestra en la Tabla 2. El mayor espesor de la capa se debe a que la difusividad del boro en el acero aumenta con la temperatura. En el proceso con la mezcla de bórax, ácido bórico y carburo de silicio la capa de boruros se obtuvo en todo el rango de temperaturas.

\begin{tabular}{|r|r|}
\hline Tiempo (h) & Espesor de la capa $(\boldsymbol{\mu m})$ \\
\hline 2 & $12.35 \pm 1.53$ \\
\hline 3 & $20.47 \pm 2.02$ \\
\hline 3.5 & $23.60 \pm 2.04$ \\
\hline 4 & $26.48 \pm 1.85$ \\
\hline
\end{tabular}

Tabla 2 Resultados del espesor de la capa del proceso de borurizado con bórax a $1273 \mathrm{~K}$

Fuente: elaboración propia [Excel]
Microscopía de Fuerza Atómica (AFM)

Las imágenes de AFM de las muestras de este estudio son las boruradas a $1273 \mathrm{~K}$ (Figura 2) durante 2 y $3,3.5$ y 4 horas.

Los resultados de la raíz cuadrada de la rugosidad $\left(R_{\mathrm{ms}}\right)$ se muestra en la Tabla 3, en los resultados no se observó un patrón de comportamiento para todos los tiempos y temperaturas. Los cambios en la rugosidad superficial fueron resultado de la formación de la capa de boruro; sin embargo, la disminución en la rugosidad superficial de los sustratos tratados termoquímicamente puede ser consecuencia de que los cristales de boruro saturaron las zonas ásperas de las muestras convirtiendo la superficie irregular en una más lisa.

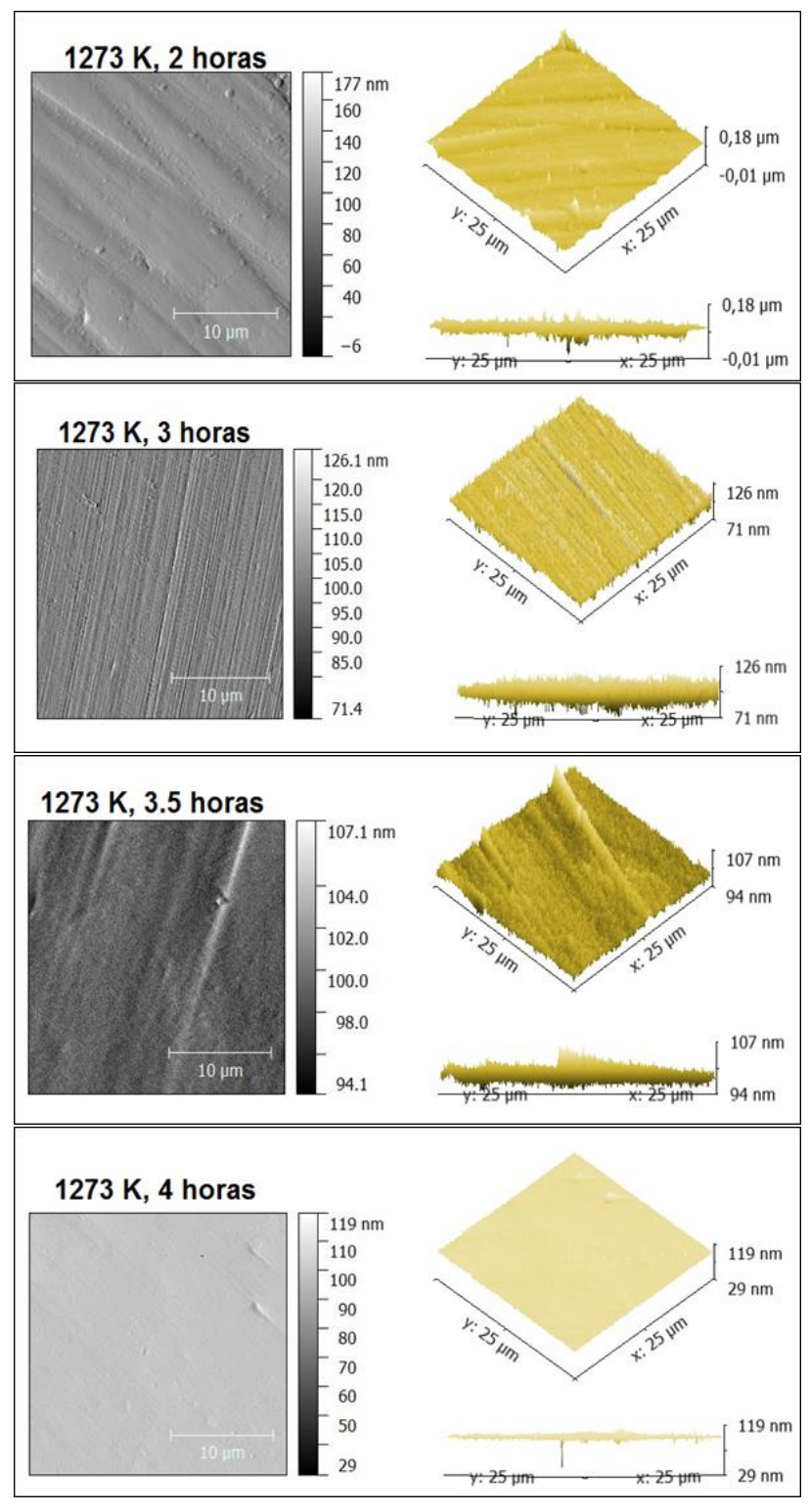

Figura 2 Imágenes del análisis por AFM del acero DIN UC1 tratado termoquímicamente por borurización a $1273 \mathrm{~K}$

Fuente: elaboración propia [AFM]

MARTÍNEZ-VÁZQUEZ, J. Merced, PÉREZ-PÉREZ, Arnulfo, RODRÍGUEZ-ORTIZ, Gabriel y BAÑOS-LÓPEZ, Esperanza. Análisis experimental y teórico del ensayo de adherencia capa sustrato en un acero DIN UC1 tratado termoquímicamente por borurización. Revista de Innovación Sistemática. 2020 


\begin{tabular}{|l|c|c|c|c|}
\hline \multicolumn{1}{|c}{ Parámetros de la muestra } & \multicolumn{5}{c|}{ Borurización a 1273 K } \\
& $\mathbf{2 h}$ & $\mathbf{3 h}$ & $\mathbf{3 . 5 h}$ & $\mathbf{4 h}$ \\
\hline Rugosidad Promedio $\left(\mathrm{R}_{\mathrm{a}}\right)[\mathrm{nm}]$ & 6.065 & 3.945 & 0.836 & 1.106 \\
\hline $\begin{array}{l}\text { Raíz cuadrada de la rugosidad }\left(\mathrm{R}_{\mathrm{ms}}\right) \\
{[\mathrm{nm}]}\end{array}$ & 8.521 & 5.103 & 1.098 & 1.553 \\
\hline $\begin{array}{l}\text { Espesor de la capa } \\
(\mu \mathrm{m})\end{array}$ & 12.35 & 20.47 & 23.6 & 26.48 \\
\hline
\end{tabular}

Tabla 3 Resultados de la rugosidad de las muestras de este estudio

Fuente: elaboración propia [Excel]

Prueba de adherencia y simulación en COMSOL®

Las imágenes obtenidas de la simulación con COMSOL® de la tensión de Von Mises al aplicar una carga de $150 \mathrm{~kg}$ sobre la capa, se muestran en la Figuras 3.

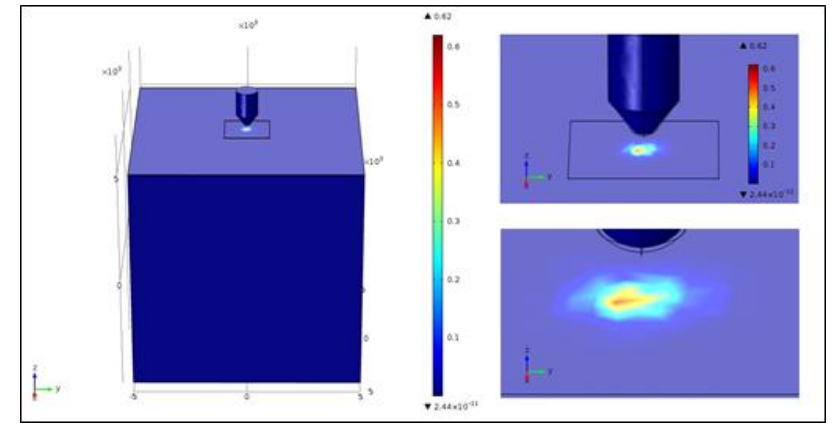

Figura 3 Resultados de la simulación de la prueba de adherencia para el acero DIN UC1 con un espesor de capa de $26.48 \mu \mathrm{m}$

Fuente: elaboración propia [COMSOL]

Las imágenes experimentales y simuladas de la tensión de Von Mises al aplicar una carga de $150 \mathrm{~kg}$ sobre la capa del acero DIN UC1 se muestran en las Figura 4, Figura 5, Figura 6 y Figura 7.
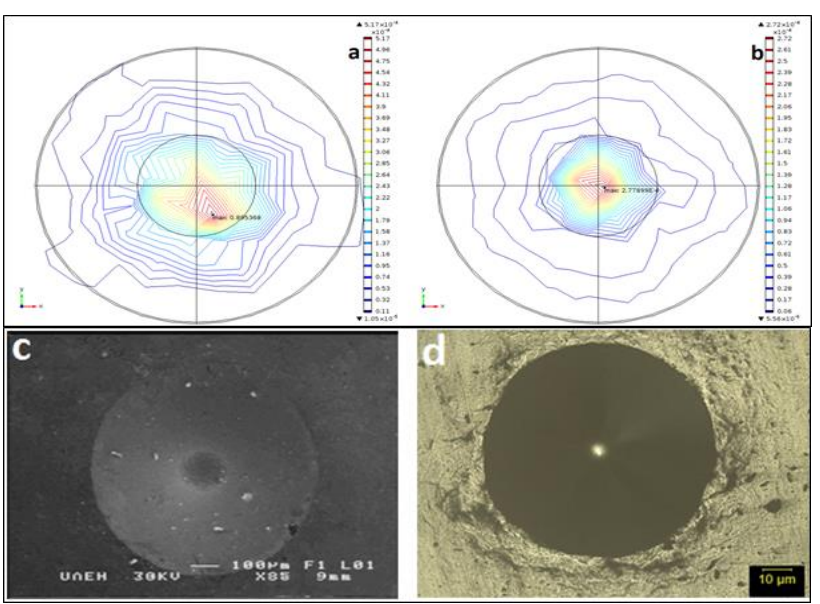

Figura 4 Resultados de la simulación de la prueba de adherencia para el acero DIN UC1 tratado a $1273 \mathrm{~K}$ durante 2 horas con un espesor de capa de $12.35 \mu \mathrm{m}$; a) tensión de Von Mises, y b) desplazamiento del material, resultados del ensayo c) micrografía por MEB y d) micrografía por MO de la indentación

Fuente: elaboración propia [COMSOL
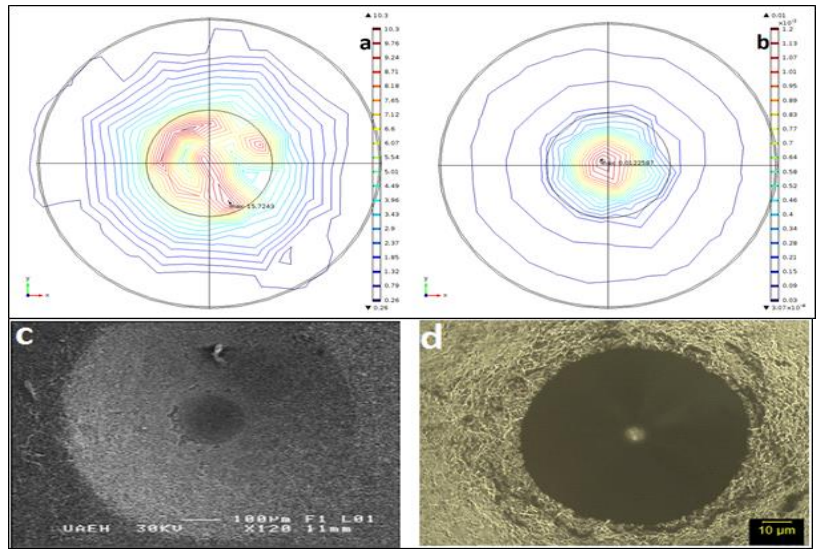

Figura 5 Resultados de la simulación de la prueba de adherencia para el acero DIN UC1 tratado a $1273 \mathrm{~K}$ durante 3 horas con un espesor de capa de $20.47 \mu \mathrm{m}$; (a) huella de indentación, (b) tensión de Von Mises en la huella, resultados del ensayo (c) micrografía por MEB y (d) micrografía por MO

Fuente: elaboración propia [COMSOL]

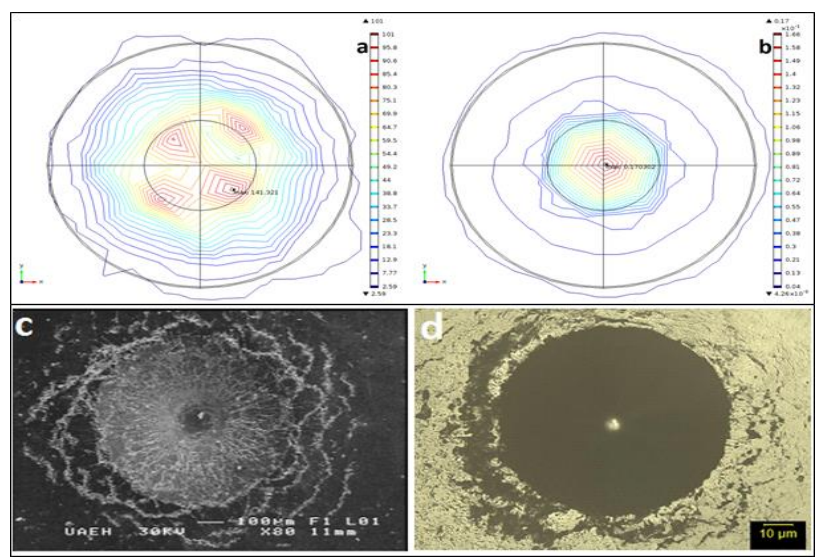

Figura 6 Resultados de la simulación de la prueba de adherencia para el acero DIN UC1 tratado a $1273 \mathrm{~K}$ durante 3.5 horas con un espesor de capa de $23.30 \mu \mathrm{m}$; (a) huella de indentación, (b) tensión de Von Mises en la huella, resultados del ensayo (c) micrografía por MEB y (d) micrografía por MO

Fuente: elaboración propia [COMSOL]

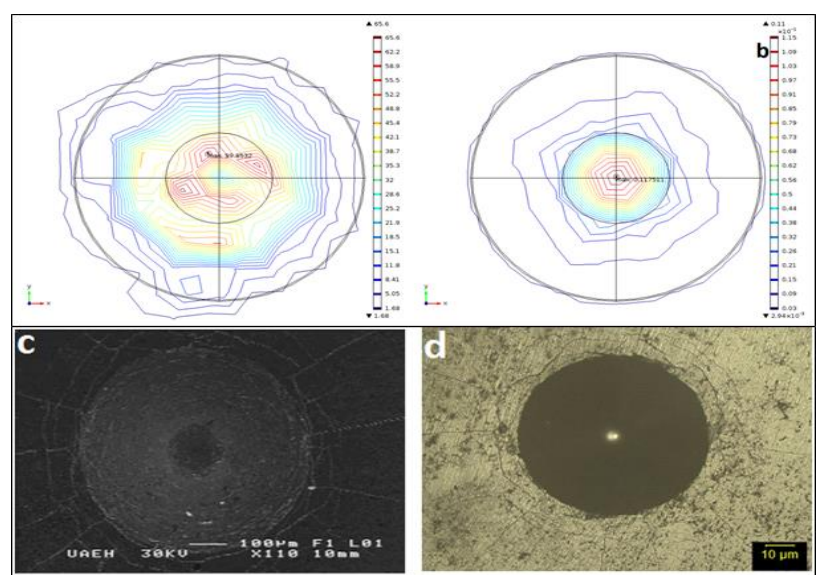

Figura 7 Resultados de la simulación de la prueba de adherencia para el acero DIN UC1 tratado a $1273 \mathrm{~K}$ durante 4 horas con un espesor de capa de $26.48 \mu \mathrm{m}$; (a) huella de indentación, (b) tensión de Von Mises en la huella, resultados del ensayo (c) micrografía por MEB y (d) micrografía por MO

Fuente: elaboración propia [COMSOL]

MARTÍNEZ-VÁZQUEZ, J. Merced, PÉREZ-PÉREZ, Arnulfo, RODRÍGUEZ-ORTIZ, Gabriel y BAÑOS-LÓPEZ, Esperanza. Análisis experimental y teórico del ensayo de adherencia capa sustrato en un acero DIN UC1 tratado termoquímicamente por borurización. Revista de Innovación Sistemática. 2020 
La tensión de Von Mises, la tensión máxima en la huella y el desplazamiento de la capa sobre el sustrato en la dirección perpendicular al contacto se muestran en la Tabla 4.

\begin{tabular}{|r|r|r|r|r|}
\hline $\begin{array}{c}\text { Espesor } \\
\text { de la } \\
\text { capa } \\
(\boldsymbol{\mu m})\end{array}$ & $\begin{array}{c}\text { Tensión } \\
\text { de von } \\
\text { Mises } \\
(\mathbf{M P a})\end{array}$ & $\begin{array}{c}\text { Tensión } \\
\text { máxima } \\
\text { en la } \\
\text { huella } \\
(\mathbf{M P a})\end{array}$ & \multicolumn{2}{c|}{$\begin{array}{c}\text { Desplazamiento } \\
\text { máximo de la capa en } \\
\text { la dirección }\end{array}$} \\
\hline 12.35 & 0.62 & 0.6 & 0.000432 & 0.000311 \\
\hline 20.47 & 0.8 & 100 & 0.04 & 0.00553 \\
\hline 23.30 & 15.5 & 1000 & 2.21 & 0.13 \\
\hline 26.48 & 123 & 100 & 0.21 & 0.02 \\
\hline
\end{tabular}

Tabla 4 Resultados de la Simulación Fuente: elaboración propia [Excel]

La huella de la indentación simulada tuvo una longitud (considerando la zona de las grietas) entre 2000-2150 $\mu \mathrm{m}$ en todos los espesores de capa. La influencia del espesor de la capa se observó en el agrietamiento alrededor de la huella, al espesor de $23.30 \mu \mathrm{m}$, las microgrietas de la capa de boruro de hierro aumentaron y el desplazamiento del material fue mayor $(2.21$ y $0.13 \mu \mathrm{m}$ en la dirección x e y, respectivamente), y por consecuencia la tensión de Von Mises en la capa de boruro fue mayor (1000 MPa).

En los espesores de capa menor (12.35 y $20.47 \mathrm{~mm}$ ) fue donde los valores de la tensión de Von Mises fueron menores, 0.62 y $0.80 \mathrm{MPa}$, respectivamente. En el espesor de capa mayor de $26.48 \mathrm{~mm}$, la tensión de Von Mises disminuyó nuevamente, con respecto al espesor anterior, y por lo tanto también disminuyó el desplazamiento en cada dirección. En todos los casos, se observa solo la formación de microgrietas, Figura 4(c y d), Figura 5(c y d), Figura 6(c y d) y Figura 7(c y d), no existe delaminación aún en aquel espesor donde la tensión fue mayor; por lo que la adherencia capasustrato es aceptable.

\section{Conclusiones}

La geometría de contacto en forma de bola del indentador Rockwell C, en combinación con la transferencia de carga, induce tensiones de corte en los extremos en la interfase. Los recubrimientos con buena adherencia soportan estas tensiones de corte y evitan la delaminación radial. La delaminación en las proximidades de la huella indica una adhesión interfacial pobre.
Las grietas radiales y poca delaminación demuestran un revestimiento fuertemente adherente, pero frágil.

Los resultados de AFM mostraron, una disminución en la $\mathrm{R}_{\mathrm{ms}}$ conforme aumentó el tiempo del tratamiento, en $1273 \mathrm{~K}$, la rugosidad estuvo en $6.065 \mathrm{~nm}$ ( 2 horas) y $1.106 \mathrm{~nm}$ (4 horas).

La simulación de la prueba de adherencia HRC basada en la norma VDI 1398; se observó para el acero DIN UC1, que en los espesores de capa menor (12.35 y $20.47 \mu \mathrm{m})$ los valores de la tensión de Von Mises fueron pequeños, 0.62 y $0.80 \mathrm{MPa}$, respectivamente, que se observó también para un espesor de capa de $26.48 \mu \mathrm{m}$, por consiguiente, también disminuyó el desplazamiento en cada dirección. En todos los casos, se observa solo la formación de microgrietas, no existe delaminación aún en aquel espesor donde la tensión fue mayor; por lo que la adherencia capa - sustrato es aceptable.

La pérdida de material reduce la capacidad de carga y la adherencia provocando un aumento en la fricción debido a la generación de residuos, por lo que, sobre la base de los datos disponibles, existe información para suponer que la fricción por deslizamiento se relaciona con velocidades de desgaste más bajas debido al empleo de recubrimientos en los que la adherencia juega un papel relevante.

Por lo tanto, para una aplicación industrial del acero DIN UC1 tratado termoquímicamente por borurización se recomiendan espesores de capa hasta $37.8 \mu \mathrm{m}$.

\section{Agradecimientos}

Los autores agradecen el apoyo de PRODEP, la UPJR y la UAEH para la realización de este trabajo.

\section{Referencias}

[1] Bataev I. A., Golkovskii M. G., Bataev A. A., Losinskaya A. A., Dostovalov R. A., Popelyukh A. I. \& Drobyaz E. A. (2014). "Surface hardening of steels with carbon by non-vacuum. Surface and Coatings, 242, 164-169. doi: j.surfcoat.2014.01.038 
[2] Reza Bateni M., Szpunar J. A., Wangb X. \& Li D. Y. (2006). Wear and corrosion wear of medium carbon steel and 304 stainless Steel. Wear, 260, 116-122. doi: 10.1016/j.wear.2004.12.037

[3] Garcia-Bustos E., Figueroa-Guadarrama M. A., Rodríguez-Castro G. A., GómezVargas O. A., Gallardo-Hernández E. A. \& Campos-Silva I. (2013). The wear resistance of boride layers measured by the four-ball test. Surface \& Coatings Technology, 215, 241-246. doi: 10.1016/j.surfcoat.2012.08.090

[4] Becker E. P. (2004). Trends in tribological materials and engine technology. Tribology International, 37, 569-575, 2004. doi: 10.1016/j.triboint.2003.12.006

[5] Li G., Chen J. \& Guan D., (2010). Friction and wear behaviors of nanocrystalline surface layer of medium carbon steel. Tribology International, 43, 2216-2221. doi: 10.1016/j.triboint.2010.07.004

[6] Tabur M., Izciler M., Gul F. \& Karacan I. (2009). Abrasive wear behavior of boronized AISI 8620 steel. Wear, 266, 1106-1112, $2009 . \quad$ doi: 10.1016/j.wear.2009.03.006

[7] Dorozkhin S. V. (2010). Bioceramics of calcium ortophosphates. Biomaterials, 31, 1465-1485. doi: 10.1016/j.biomaterials.2009.11.050

[8] Chong W. F. \& Chidlow S. J. (2016). Modelling adhesive contact problems involving a layered elastic solid and cylindrical indenter using Lennard Jones potential. Mechanics of Materials, 84, 111. doi: 10.1016/j.mechmat.2015.01.006

[9] Márquez-Herrera A., Fernández-Muñoz J. L., Zapata-Torres M., Melendez-Lira M. \&. Cruz-Alcantar P. (2014). $\mathrm{Fe}_{2} \mathrm{~B}$ coating on ASTM A-36 steel surfaces and its evaluation of hardness and corrosion resistance. Surface \& Coatings Technology, 254, 433-439. doi: 10.1016/j.surfcoat.2014.07.001
[10] Kartal G-, Timur S., Sista V., Eryilmaz O. L. \& Erdemir A. (2011). The growth of single $\mathrm{Fe}_{2} \mathrm{~B}$ phase on low carbon steel via phase homogenization in electrochemical boriding (PHEB). Surface \& Coatings Technology, 206, 2005-2011. doi: 10.1016/j.surfcoat.2011.08.049

[11] Kulka M., Pertek A. \& Makuch N. (2011). The importance of carbon concentrationdepth profile beneath iron borides for lowcycle fatigue strength. Materials Science and Engineering, 528, 8641-8650. doi: 10.1016/j.msea.2011.08.018

[12] Vidakis N., Antoniadis A. \& Bilalis N. (2003). The VDI 3198 indentation test evaluation of a reliable qualitative control for layered compounds. Journal of Materials Processing Technology, 1, 481485, 2003. doi: 10.1016/S09240136(03)00300-5

[13] Holmberg K. et al, (1998). Coatings tribology-contact mechanisms and surface design. Tribology International, 31, 107-120. doi: 10.1016/S0301679X(98)00013-9

[14] Findik F. (2014). Latest progress on tribological properties of industrial Materials. Materials and Design, 57, 218244. doi: 10.1016/j.matdes.2013.12.028

[15] ASM, Handbook Volume 4 (1994). Surface Engineering, USA: ASM International. ISBN: 978-0-87170-384-2

[16] Martínez-Vázquez, J. M., Vargas-Ramírez M., Cortés-Campos M. L. \& HortelanoCapetillo J. G. (2019). Cálculo de la energía de activación de la difusión de boro en la capa de $\mathrm{Fe}_{2} \mathrm{~B}$ en la borurización por empaquetamiento de los aceros SAE 1050 y DIN UC1. Revista de Energía Química y Física, 6, 8-17. doi: 10.35429/JCPE.2019.18.6.8.17

[17] Bouarour B., Keddam M., \& Allaoui O. (2014). Estimation of the effective borondiffusion coefficient in the $\mathrm{Fe}_{2} \mathrm{~B}$ layers grown on gray cast iron. MTAEC9, 48, 515-520. UDK 546.271:532.72 
[18] Carbucicchio M. \& Palombarini G. (2004). Mechanism of thermochemical growth of iron borides on iron. Journal of Materials Sciences, 39, 933-937. doi: SC.0000012924.74578.87

[19] Keddam M. (2004). A kinetic model for the borided layers by the paste-boriding process. Applied Surface Science, 236, 451-455. doi: 2004.05.141

[20] Keddam M., Kulka M., Makuch N., Pertek A. \& Maldzinski L. (2014). A kinetic model for estimating the boron activation energies in the $\mathrm{FeB}$ and $\mathrm{Fe} 2 \mathrm{~B}$ layers during the gas boriding of Armco iron: Effect of boride incubation times. Applied Surface Science, 298, 155-163. doi: 2014.01.151. 